\title{
Cognitive function in treated HIV patients
}

This article was published in the following Dove Press journal:

Neurobehavioral HIV Medicine

12 November 2010

Number of times this article has been viewed

\section{Valerio Tozzi \\ Pietro Balestra \\ Raffaella Libertone \\ Andrea Antinori}

Lazzaro Spallanzani National Institute for Infectious Diseases, Rome, Italy
Correspondence: Valerio Tozzi Lazzaro Spallanzani National Institute for Infectious Diseases, Via Portuense 270, 00149 Rome, Italy

Tel +390655170427

Fax +390655I70405

Email valerio.tozzi@inmi.it
Abstract: Although highly active antiretroviral therapy (HAART) has reduced the incidence of HIV-associated dementia, the overall prevalence of HIV-associated neurocognitive disorders (HAND) has increased. Since treatment and prevention of HAND are becoming an increasing concern, management strategies for cognitive impairment in patients living with HIV are expected to play an important role in the near future. This review summarizes the existing literature on studies investigating cognitive functions in patients receiving antiretroviral (ARV) treatment. Most studies indicate that HAART use results in improvement of neurocognitive functions, yet milder forms of HAND are not only prevalent, but also incident in patients with persistently undetectable plasma HIV RNA. We performed a systematic review on all studies performed in patients receiving ARV treatment that included neurocognitive evaluations as study end points. Thirty-six studies were examined. Study methodology varied from cross-sectional to doubleblind, randomized, controlled designs. Aside from historic zidovudine monotherapy trials, in most studies, ARV schemes varied considerably, and in some cases, ARV regimens were not reported in detail. Only a few articles included virological studies on cerebrospinal fluid. Study duration was $<2$ years in most cases. Differences in study design and presence of study limitations may account for difficulties in understanding the impact of HAART on cognition and in evaluating the effect of an individual agent or ARV regimen on cognitive functioning. The aim of the present article is to provide HIV clinicians with a comprehensive review of recent achievements and future prospects for ARV treatment and prevention of cognitive dysfunctions in HIV-infected patients.

Keywords: highly active antiretroviral therapy, neurocognitive disorders, HIV dementia, central nervous system

\section{Introduction to cognitive impairment in HIV patients}

The central nervous system (CNS) is a major target of HIV-1 infection. HIV invades the CNS during the primary infection period and establishes a protected viral reservoir. In a consistent proportion of patients, chronic CNS HIV infection can lead to neurodegenerative complications that have recently been characterized as HIV-associated neurocognitive disorders (HAND). HAND defines three categories of dysfunction: asymptomatic neurocognitive impairment (ANI), mild neurocognitive disorder (MND), and HIV-associated dementia (HAD). ${ }^{1}$

The introduction of highly active antiretroviral therapy (HAART) as a standard of care has considerably enhanced the life expectancy among HIV-infected individuals. Over the last 20 years, antiretroviral (ARV) therapy has moved from an almost 
ineffective monotherapy to combination multidrug regimens able to suppress plasma viral load to undetectable levels in most HIV-infected patients. Consequently, the natural history of HIV infection has been changed into a manageable chronic disease requiring long-term ARV treatment. However, despite this success, there are a number of important issues that needs to be addressed. Nowadays, the incidence of the most severe form of HAND and HAD has declined..$^{2-4}$ However, HAND represents an unresolved clinical issue. First of all, while HAD has become less frequent, MND and ANI may persist in a relevant proportion of patients. ${ }^{5-7}$ Second, consistent literature has demonstrated strong relationships between HAND and employment, medication adherence, mood, fatigue, interpersonal functioning, quality of life, survival, and use of healthcare resources. ${ }^{8-11}$ Finally, because the HIV-infected population is aging, patients are exposed to a growing number of comorbidities with negative impact on cognitive functioning. ${ }^{12}$ Thus, identifying patients at risk of HAND and developing treatment strategies able to prevent and effectively treat HIV-associated cognitive dysfunctions should become a priority.

This review summarizes the existing literature on prevalence, clinical presentation, and treatment strategies of HAND.

\section{Methods}

\section{Search strategy}

The search was conducted using different strategies. First, a literature search was performed using the PubMed database. Search terms that might indicate cognitive function (cognitive impairment, HIV-associated dementia, AIDS dementia complex, cognition, and neuropsychological tests) and ARV treatment (antiretroviral therapy, treatment, and HAART) were used as keywords. Second, the names of key researchers in the topic were used as authors for PubMed database searches. Third, the reference sections of relevant studies on the topic were screened.

\section{Eligibility criteria, inclusion and exclusion criteria}

All studies assessing neuropsychological (NP) measures as primary or secondary end points in patients receiving ARV therapy were included. We defined ARV therapy as any treatment with ARV drugs, either alone like zidovudine monotherapy or in combination. We defined NP measures as a NP battery that included at least three NP tests. The time period covered was from 1987 to June 2010. Studies on children and adolescents were excluded.

\section{Clinical presentation and prevalence of cognitive impairment in HIV Clinical presentation}

The diagnostic criteria for HAND were updated in $2007 .{ }^{1}$ These criteria recognize 3 categories of HAND: the asymptomatic form (ANI), the mild form (MND), and the severe form (HAD). Patients with ANI show an acquired impairment in cognitive functioning, documented by detailed NP testing exploring at least 5 cognitive domains, in at least 2 domains. The cognitive impairment is asymptomatic and does not interfere with everyday functioning.

HAD lies on the opposite side of the clinical spectrum. $\mathrm{HAD}$ is among the most devastating consequences of HIV infection due to its progressive impact on everyday life. It is characterized by moderate to severe neurocognitive impairment, affecting at least 2 cognitive domains. ${ }^{1}$ Early clinical features include a combination of a triad of manifestations: cognitive (forgetfulness, poor concentration, and attention deficits), motor (tremor, ataxia, and spasticity), and behavioral (agitation and apathy). ${ }^{9}$ In patients with HAD, the impairment is moderate to severe and produces a marked interface with daily functioning (work, home life, and social activities). ${ }^{1}$ Later in the course of the disease, global dementia, paraplegia, and mutism may occur. Typically, in untreated HIV-infected patients, HAD evolves insidiously over a period of weeks or months. ${ }^{9}$

The intermediate form of HAND is MND. MND is often an antecedent syndrome and can precede the onset of franc dementia. Clinical features of MND are similar to HAD, although with less severe signs and symptoms. The cognitive impairment, involving at least 2 cognitive domains, produces a mild interface in daily functioning. ${ }^{1}$

\section{Incidence and prevalence}

Epidemiological and clinical studies have provided evidence for a change in HAND in the era of HAART. Since the introduction of HAART as a standard of care, there has been a decline in the incidence of HAD as presenting manifestation of AIDS. ${ }^{2-4}$

However, despite this improvement, cognitive decline remains a common feature of HIV infection. Data from the literature consistently indicate that, compared to the pre-HAART era, the overall prevalence of HAND is still considerably high. In the early HAART era, Ferrando et $\mathrm{al}^{5}$ performed a cross-sectional study of 130 symptomatic HIV-positive patients. A battery of six NP tests assessing the domains of attention, concentration, learning, memory, psychomotor speed, and executive function was used. Among 
the 69 patients taking HAART, the overall prevalence of less severe forms of cognitive impairment was $22 \%$. Subsequent reports indicated that, compared to the pre-HAART era, the prevalence of HAD could be unchanged or even increasing. ${ }^{13,14}$ A total of $202 \mathrm{HIV}$-positive patients enrolled in the Hawaii Aging with HIV Cohort underwent a cross-sectional evaluation with a comprehensive NP battery of 12 tests assessing multiple cognitive domains. ${ }^{15}$ HAD was diagnosed in $25.2 \%$ of older ( $>50$ years) and in $13.7 \%$ of younger subjects, and mild forms of cognitive impairment were observed in $44.7 \%$ of older and in $26.3 \%$ of younger participants. In more recent estimates, the prevalence of HAND, especially in its less severe forms of MND and ANI, is still considerably high. Robertson et al performed a prospective cohort study among 1160 subjects enrolled in the ALLRT study. ALLRT is a prospective observational cohort enrolling patients participating in AIDS Clinical Trials Group (ACTG) who have been randomly assigned to ARV therapies, immune-based therapies, or strategies for anti-HIV interventions. Every 48 weeks, subjects performed tests to screen for HIV-related neurological diseases. A brief neurocognitive screen battery, consisting of 3 NP tests, was used. Row test scores were standardized using demographic-adjusted normative means. Relative to the normative data, 2 levels of impairment were defined: mild and mild to moderate. A total of 458 subjects out of $1160(39 \%)$ were classified as having at least mild neurocognitive impairment. Using the most conservative estimate, 304 of $1160(26 \%)$ patients had at least mild to moderate impairment. Moreover, among patients classified impaired at baseline, 56\% remained impaired at follow-up visits. Finally, of 615 subjects who were unimpaired at baseline, 128 (21\%) became impaired at subsequent visits. ${ }^{6}$ HAD was observed at similar frequencies in sub-Saharan Africa. ${ }^{16}$ Wong et al performed a cross-sectional study in an ambulatory clinic in Uganda. A battery of eight NP tests exploring 5 cognitive domains was administered to $78 \mathrm{HIV}$-infected patients. The results of NP testing of 100 HIV-negative subjects were used to construct normative data. Standardized neurologic assessment, medical history, functional status assessment, and normatively adjusted NP test scores were used to classify patients as having HIV dementia. Overall, the frequency of HIV dementia was $31 \% .{ }^{16}$ More recently, the prevalence of cognitive deficits was explored in a cohort of 200 patients with long-standing undetectable plasma HIV RNA. ${ }^{7}$ Patients with confounding comorbid conditions were excluded. Subcortical functions were evaluated using a battery of 9 tests exploring a wide range of cognitive domains. Mood disorders, behavioural changes, and functional impairment were assessed using standardized instruments. The updated research nosology for HAND was used to define patients as having ANI, MND, or HAD. ${ }^{1}$ Overall, the estimated prevalence of HAND in this cohort of HAART-treated patients with an undetectable viral load was $69 \%$. More in details, the proportion of patients with ANI, MND, and HAD was $42 \%, 28 \%$, and $4 \%$, respectively. These data indicate that although HAND without functional repercussion on daily life was the most frequent subtype of HAND, neurocognitive impairment may be present in more than $50 \%$ of patients on successful HAART.

In summary, current estimates indicate that HAND can develop in a considerable portion of HIV-infected patients despite effective viral suppression in the periphery.

\section{Risk factors}

Risk factors for HAND include current or past low CD4 cell count, ${ }^{6,15-18}$ advanced age, ${ }^{15,16,19}$ coinfection with hepatitis $\mathrm{C}$ virus, ${ }^{18,20}$ insulin resistance or diabetes, ${ }^{19}$ and cardiovascular risk factors. ${ }^{21}$ Advanced immune suppression, either current or historical, and older age are strong risk factors for HAND, being reported in most published studies. Among the 202 patients enrolled in the Hawaii Aging with HIV Cohort, HAD was more frequent in older $(>50$ years $)^{15}$ compared to younger individuals (25.2\% and $13.7 \%$, respectively; $P=0.04)$. Among older individuals, meeting HAD criteria was significantly associated with low CD4 cell count $(\mathrm{OR}=7.13 ; 95 \% \mathrm{CI}=1.8-28.2) .{ }^{15}$ Within the ALLRT study, after adjusting for race, education, age, sex, and ARV therapy, both previous and current CD4 cell count $<200 /$ uL were significantly associated with neurocognitive impairment. ${ }^{6}$ Wong et al examined frequency and risk factors for HAD in an HIV clinic in Uganda. ${ }^{16}$ At regression analysis, older age and lower CD4 cell count were significantly associated with the diagnosis of HAD. Each additional 10 years of age conferred a greater than twofold risk of $\operatorname{HAD}(P<0.05)$, and reduced levels of CD4 count (100 cells/uL decrement) was associated with a $60 \%$ increase in the odds of having HAD $(P<0.05)$. Nadir CD4 counts $<200$ cells/uL had been associated not only with a higher prevalence of neurocognitive deficits, but also with decreased functioning in selected neurocognitive areas such as attention, working memory, and executive function. ${ }^{17}$

Insulin resistance, diabetes, ${ }^{19}$ and cardiovascular risk factors may also be detrimental to neurocognitive functioning, especially in patients with high CD4 cell count. ${ }^{21}$ Wright et al assessed risk factors associated with baseline neurocognitive performance in HIV-infected patients enrolled in the SMART substudy. ${ }^{21}$ A total of 292 subjects were administered a five-test 
NP battery. Median CD4 cell count was 536/uL. Overall, 14\% of patients had neurocognitive impairment. Prior cardiovascular disease $(\mathrm{OR}=6.2 ; P=0.01)$, hypercholesterolemia $(P=0.02)$, and hypertension $(P=0.03)$ were associated with poorer neurocognitive performance, but low CD4 cell count and other risk factors for HAD were not.

\section{Role of comorbidities}

Since HIV-infected patients are living longer, they are exposed to an increasing number of comorbidities that could have a relevant impact on cognition. These include hepatitis $\mathrm{C}$, current or previous substance abuse (methamphetamine, narcotics, alcohol, and cocaine), major psychiatric disorders (bipolar illness, schizophrenia, and major depression), CNS opportunistic infections and/or tumors, major stroke, transient ischemic attacks, head injury, multiple sclerosis, and other dementing disorders (Alzheimer's disease). ${ }^{1,20,22}$ Finally, aging itself could play a role in contributing to cognitive impairment in patients with HIV infection. ${ }^{9,12,23}$

The role of comorbidities has been recently categorized as incidental, contributing, and confounding. ${ }^{1}$ An incidental comorbidity may have minor effects on NP test results, but is unlikely to cause even mild global impairment. Incidental comorbidity does not preclude diagnosis of HAD. A contributing comorbidity is likely to have at least mild effects on NP test results, but is unlikely to cause clinically significant global impairment by itself. A contributing comorbidity does not preclude diagnosis of HAD. Finally, a confounding comorbidity is more severe and is likely to have major effects on NP test results, with significant neurocognitive impairment and functional disability, or is likely to cause invalid NP test results. A confounding comorbidity precludes diagnosis of HAD.

Moreover, while comorbidities may act as confounders to accurate diagnosis of HAND, they could also have an additive effect if HAND is already present. Thus, cognitive impairment in patients on HAART may also occur as a consequence of multifactorial etiology, with neurocognitive consequences of HIV infection sometimes overlapping with aging and with comorbidities directly affecting cognition.

\section{Efficacy of commonly used treatments such as ARV therapy}

The optimal treatment for HAND has not been established, but there is strong evidence that ARV therapy can improve cognitive dysfunction. Here, we summarize the existing literature on the topic. Study design, patients' characteristics, NP test batteries, cognitive domains, ARV treatment, and main findings of studies evaluating the effects of ARV therapy on cognition are reported in Table 1.

\section{Mono-ARV therapy}

Several studies were performed with zidovudine monotherapy. The importance of these historical studies is that they first documented the beneficial effect of ARV therapy on the course of HAD. Moreover, at that time, 2 large, doubleblinded, placebo-controlled studies clearly demonstrated the superiority of zidovudine monotherapy versus placebo on end points exploring cognition. ${ }^{24,25}$ These are among the few randomized, controlled studies on HAD ever performed. Schmitt et al first demonstrated that advanced HIV-infected patients receiving zidovudine showed improved cognition, in measures exploring attention, memory, and motor functioning compared with patients receiving placebo. ${ }^{24}$ Subsequently, the same was shown in patients fulfilling the criteria for HAD. ${ }^{25}$ Finally, it was reported that in some patients, the neurological benefits associated with zidovudine use could sometimes be transient. ${ }^{26}$

\section{HAART improves neurocognitive function}

In 1998, a cross-sectional study on advanced HIV-infected patients first reported that subjects taking HAART performed significantly better on tests of attention, concentration, learning, memory, and psychomotor speed than those not taking HAART. ${ }^{5}$ A bit later, by incorporating serial NP evaluations in a randomized, multicenter trial enrolling 1031 patients, it was shown that a combination therapy of zidovudinedidanosine-nevirapine preserved or improved NP performance compared to alternating zidovudine-didanosine and zidovudine-zalcitabine combination regimens. ${ }^{8}$ Subsequently, several observational studies independently reported significant NP improvements in patients receiving protease inhibitor (PI)-based HAART (Table 1). ${ }^{27,28}$

Additional studies confirmed the finding in different populations including women ${ }^{29,30}$ and patients in sub-Saharan Africa (Table 1). ${ }^{31}$ Moreover, it was shown that the cognitive domains enhanced with HAART were psychomotor speed, verbal anterograde memory, and executive functions, thus supporting the idea that HAART could revert cognitive dysfunction by improving subcortical cognitive functions. ${ }^{32}$ To further assess the pattern and durability of neurocognitive benefits, Ferrando et al performed a prospective evaluation of 141 patients over a 2.5 -year period. They found that the impairment rate decreased from $62 \%$ to $33 \%$. Moreover, after controlling for multiple factors including the potential effects of practice, the use of potent HAART was associated 


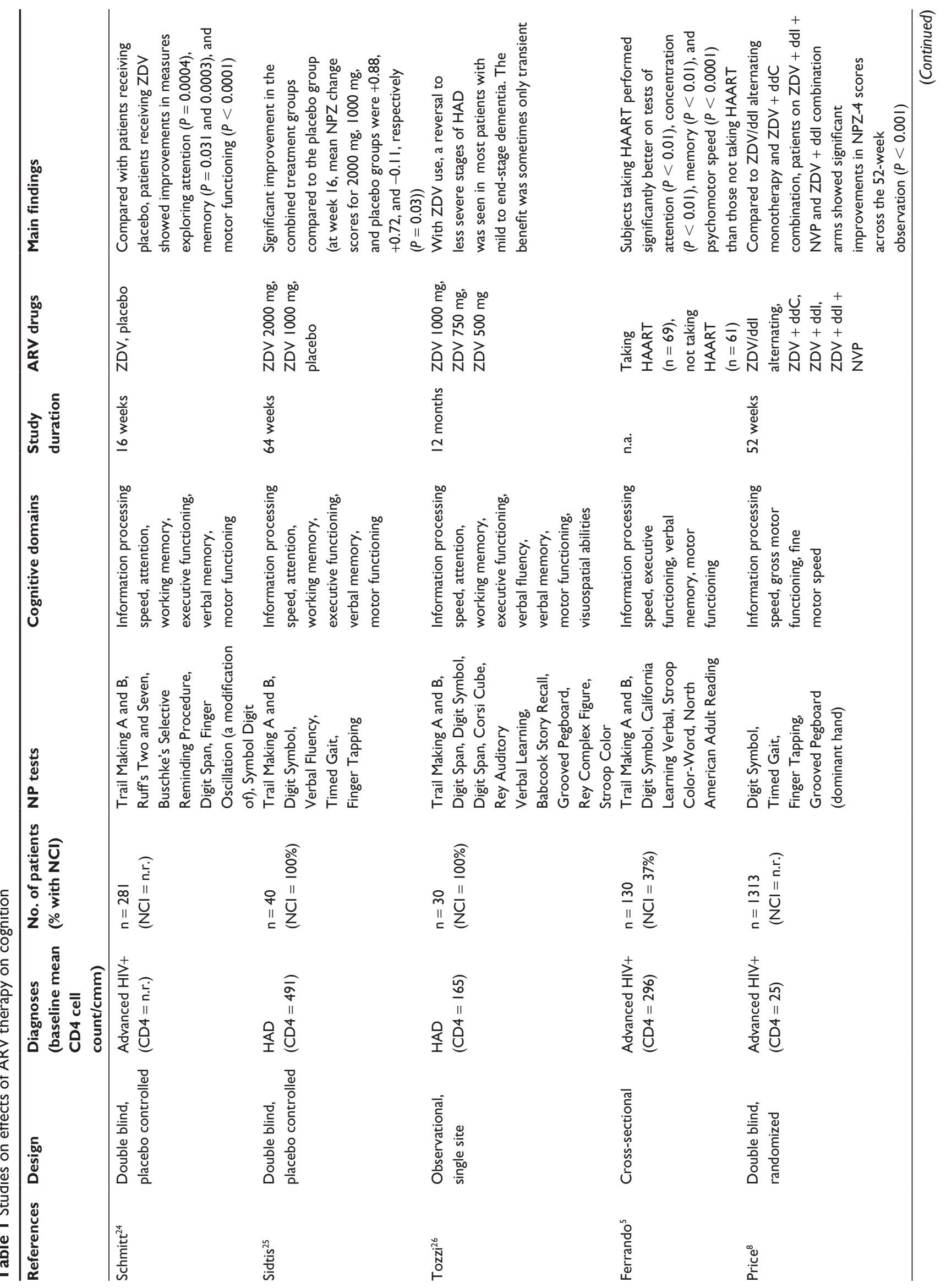




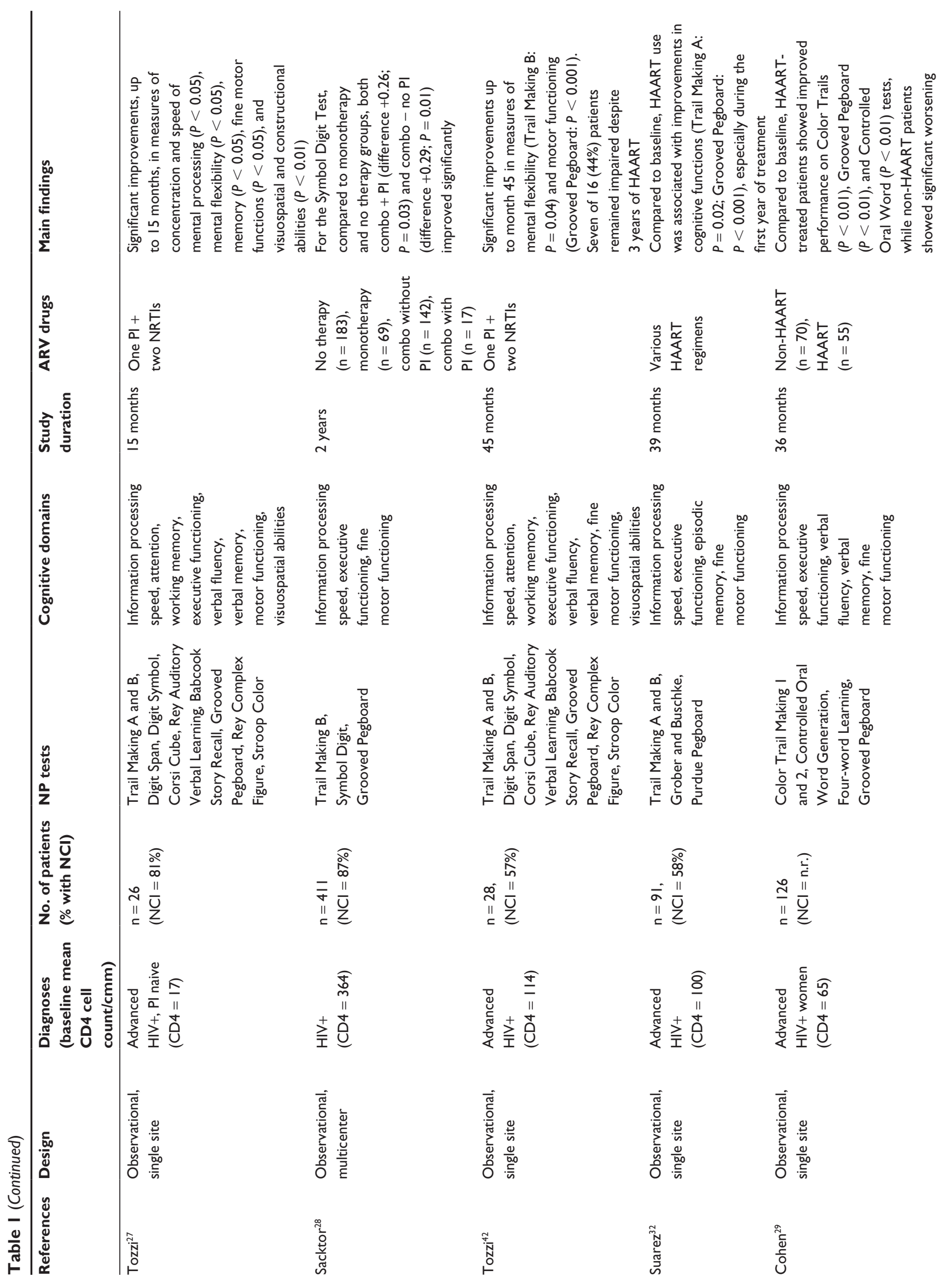



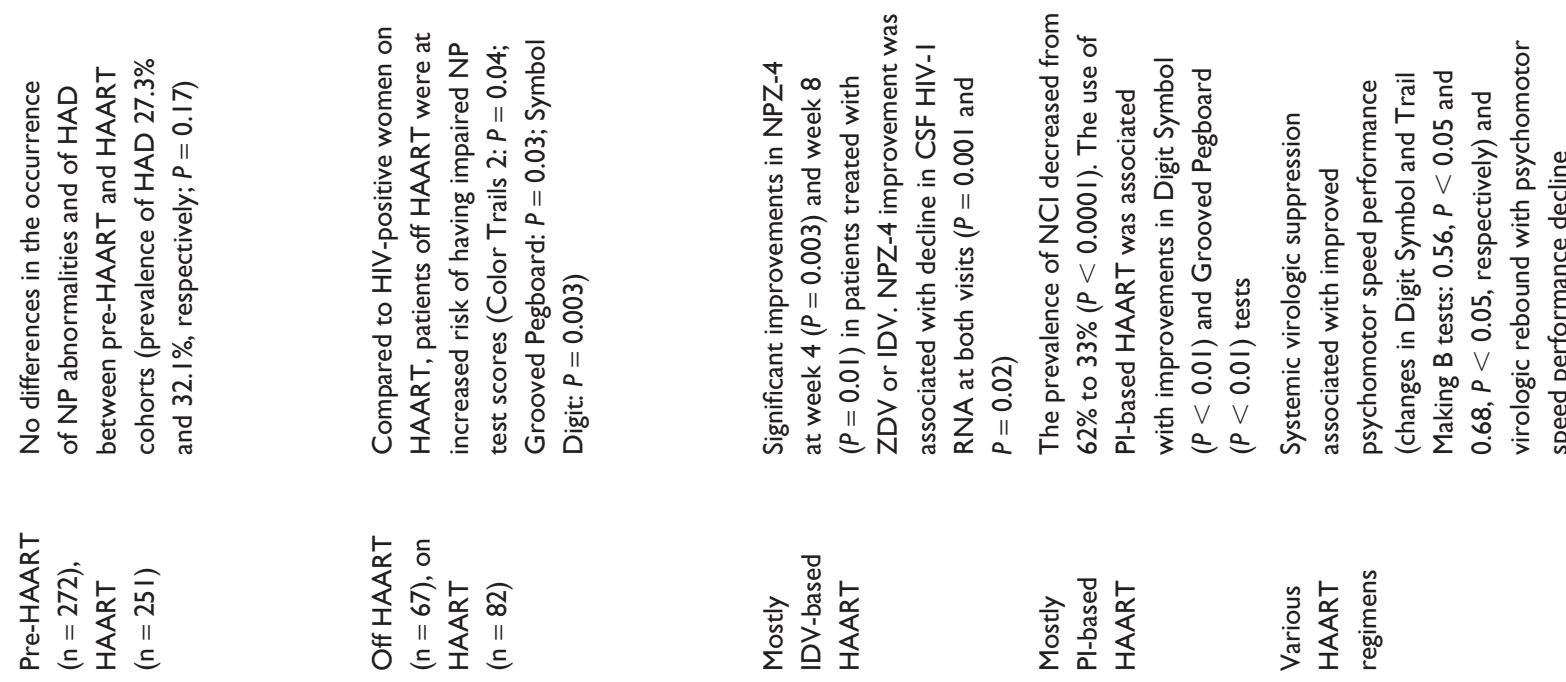

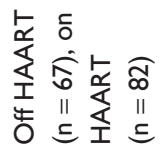

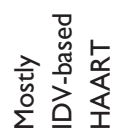

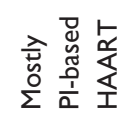

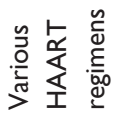

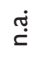

$\stackrel{d}{\check{g}}$

$\frac{y}{d}$

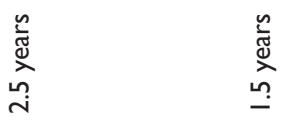
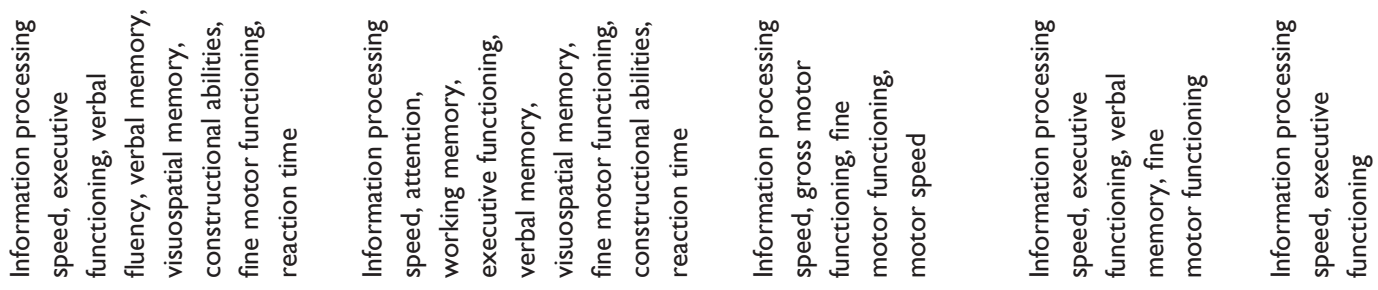

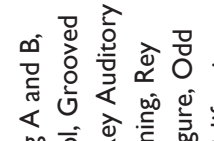

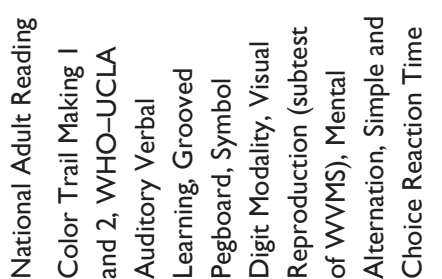

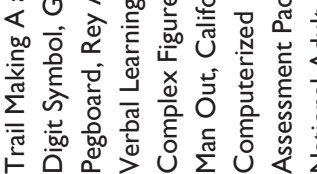

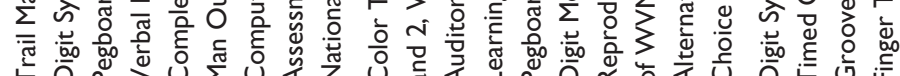
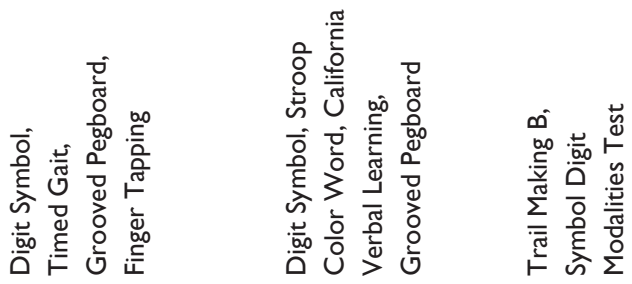

กָก

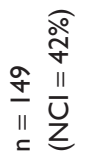

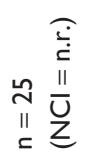

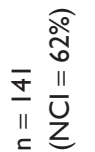

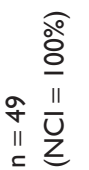

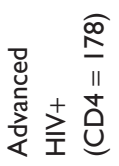

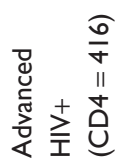

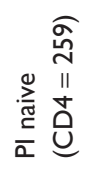

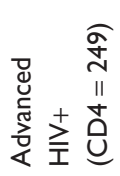

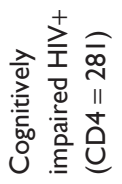

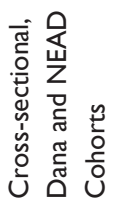
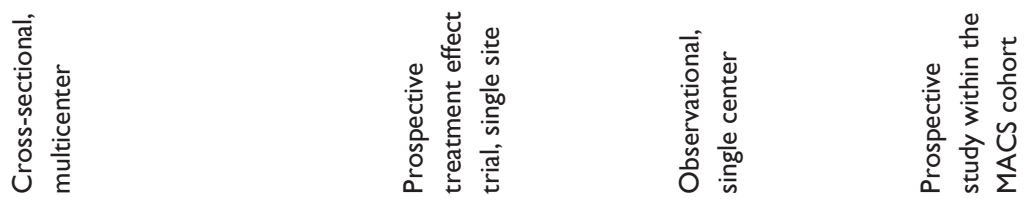

ڤั

0
0
0
$\frac{0}{0}$
$\frac{0}{0}$
$\frac{c}{x}$

$\frac{\sum^{\frac{\pi}{2}}}{\frac{\pi}{2}}$

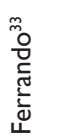

童 


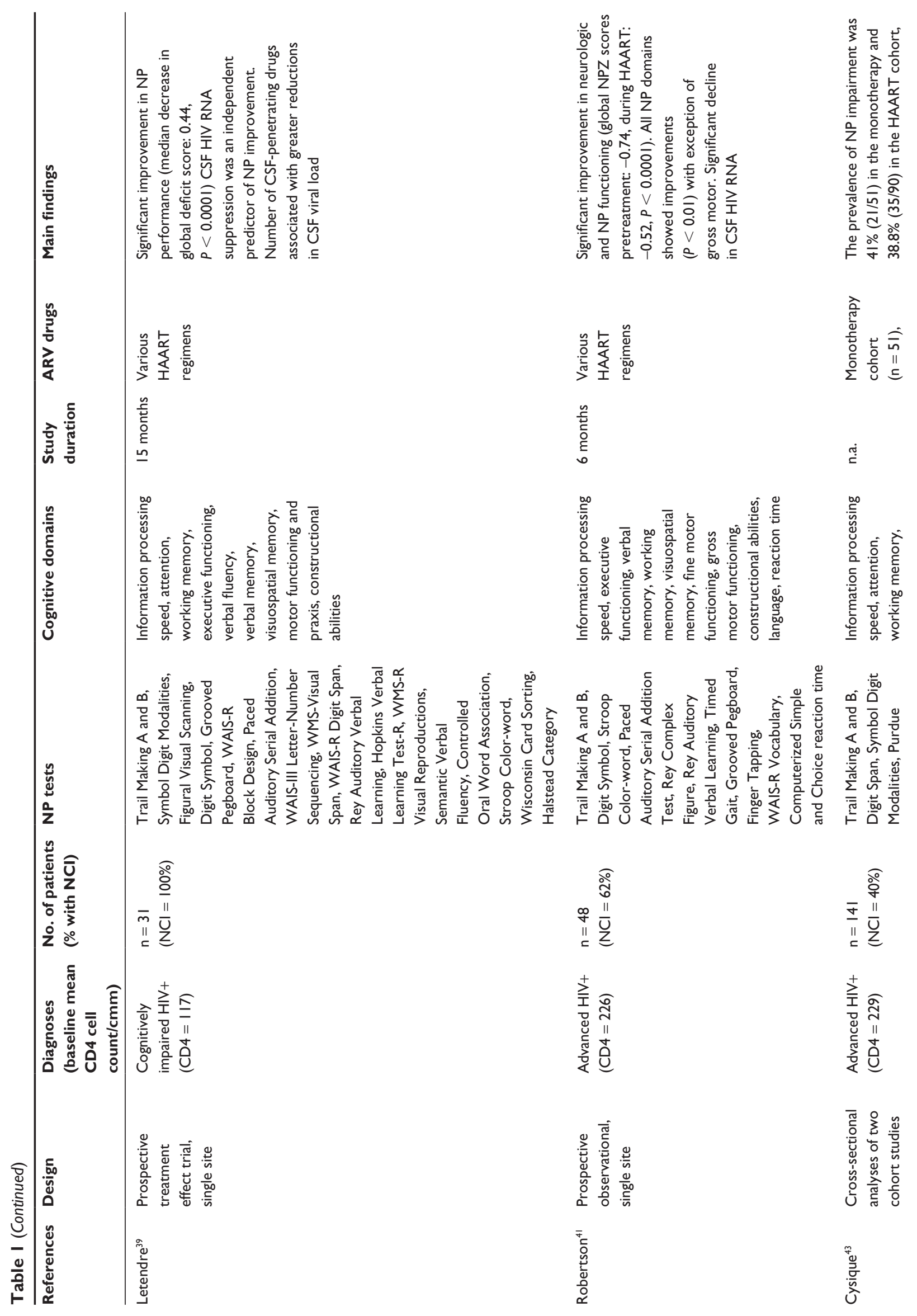




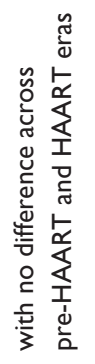
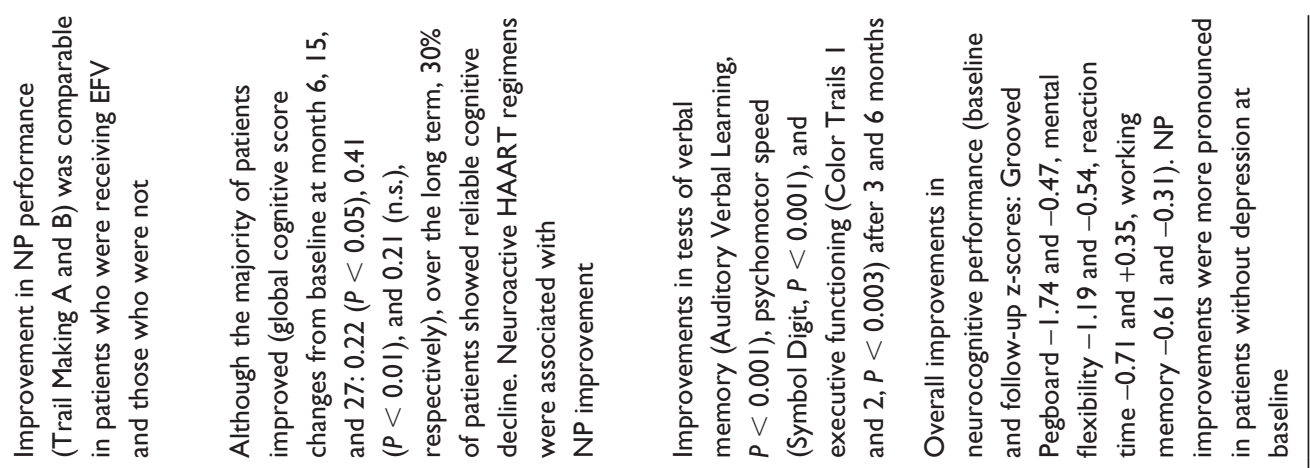

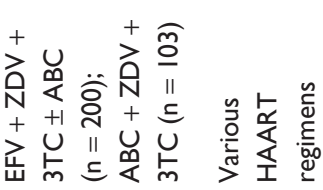

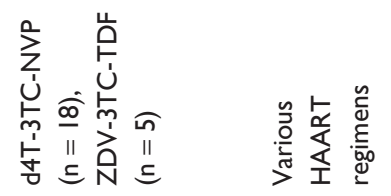
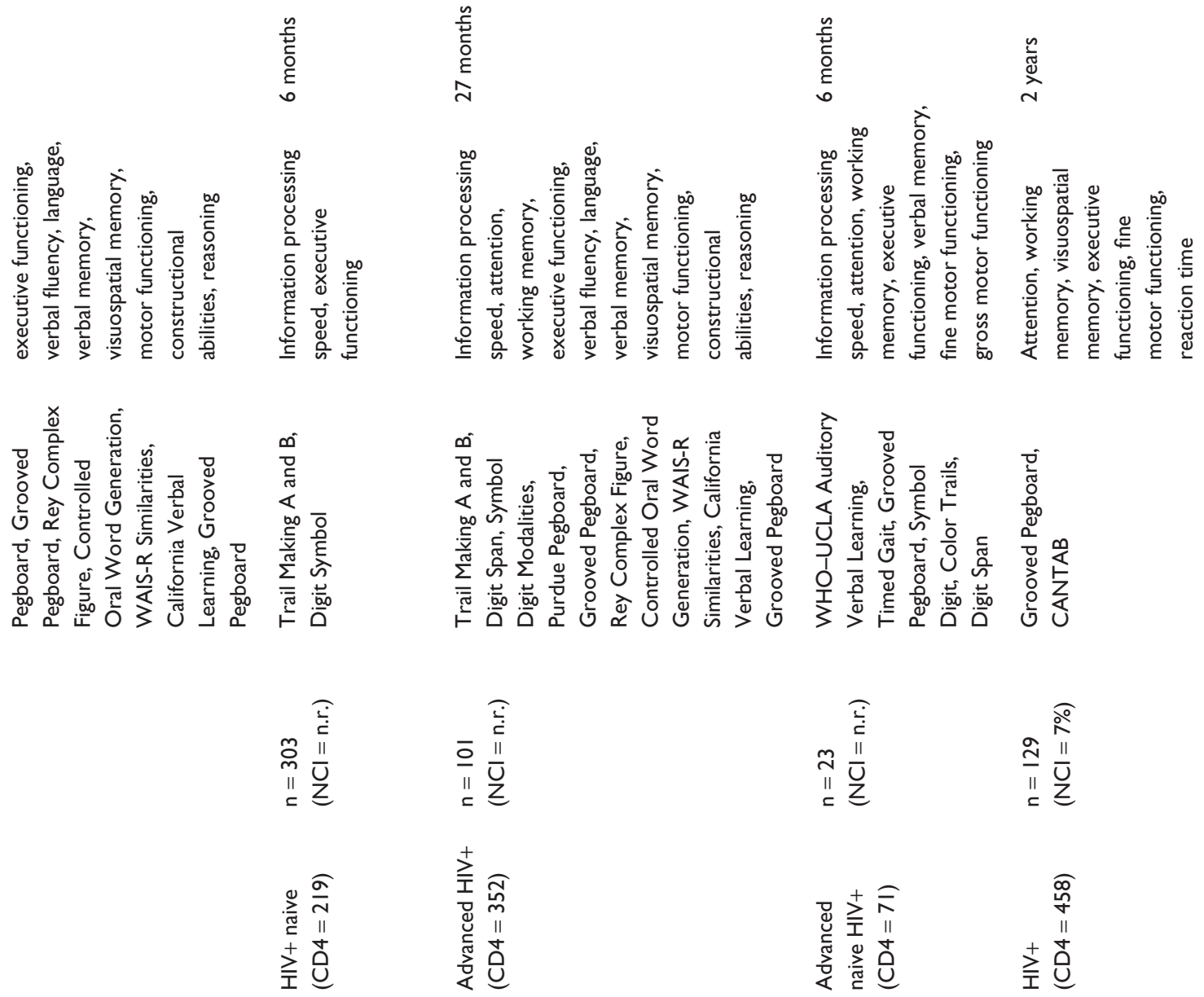

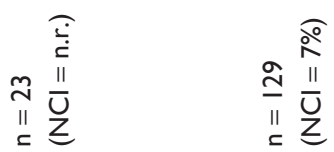
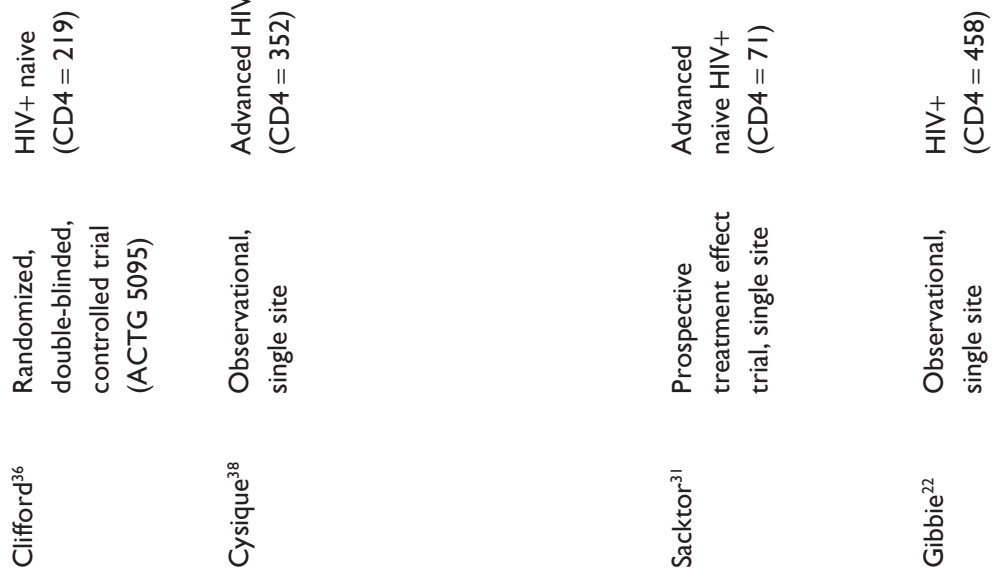


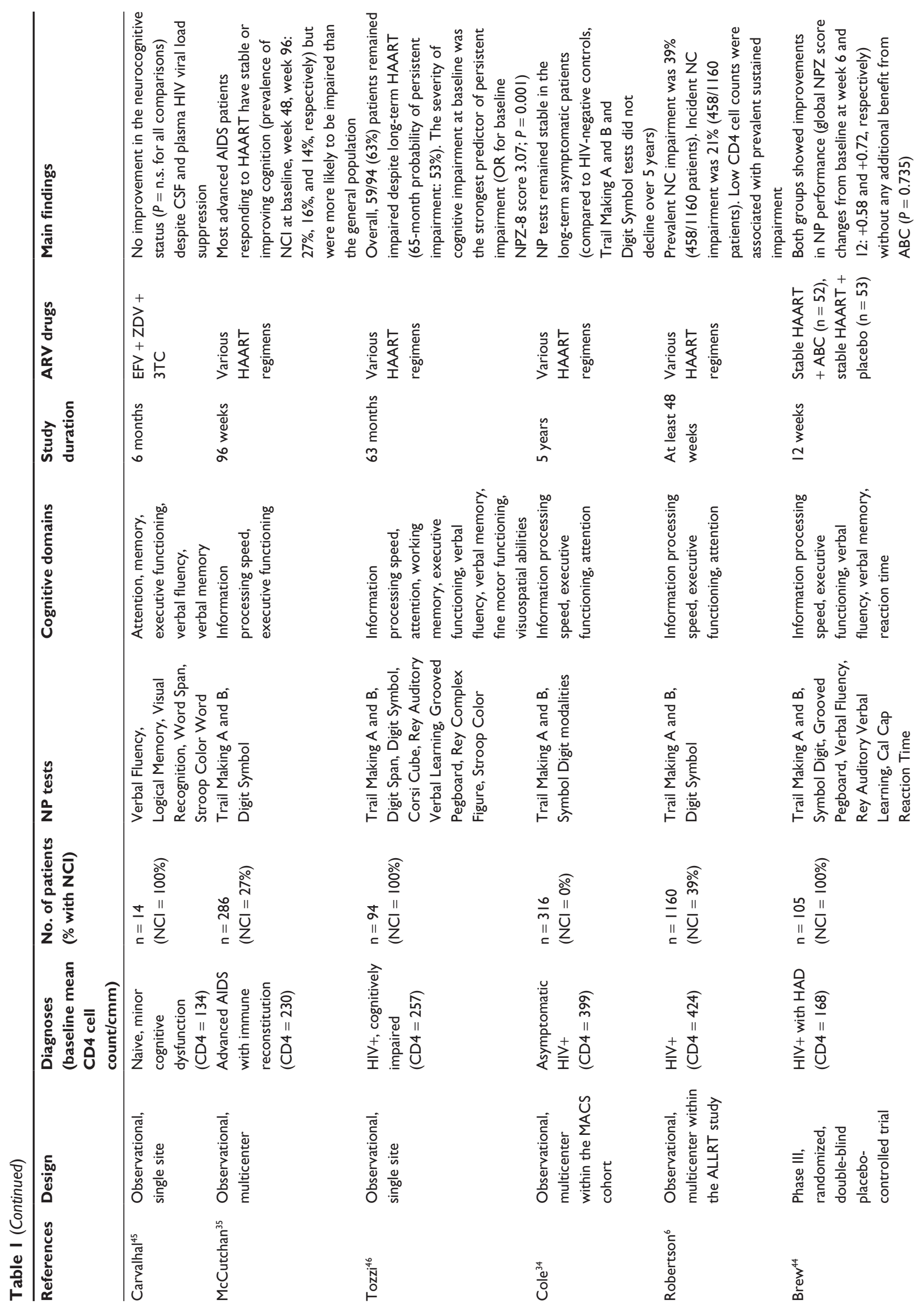



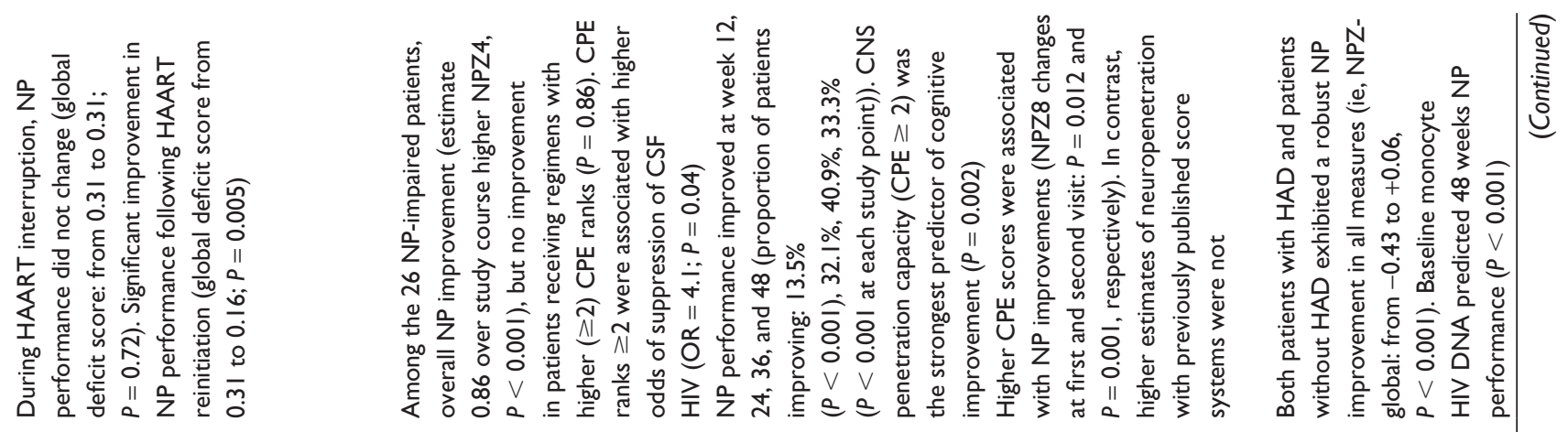

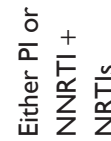

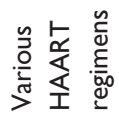

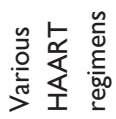

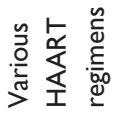

$\stackrel{n}{气}$

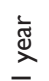

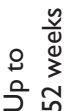

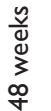

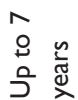

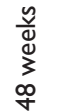
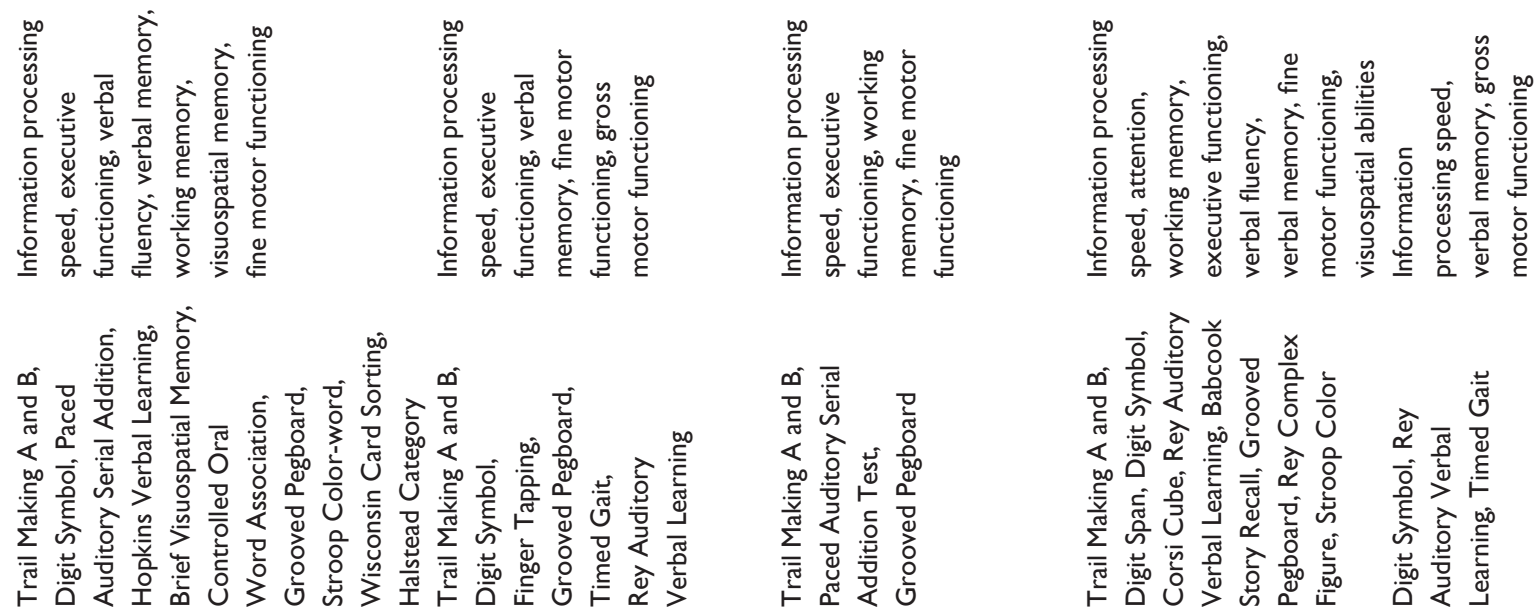

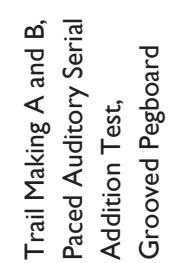
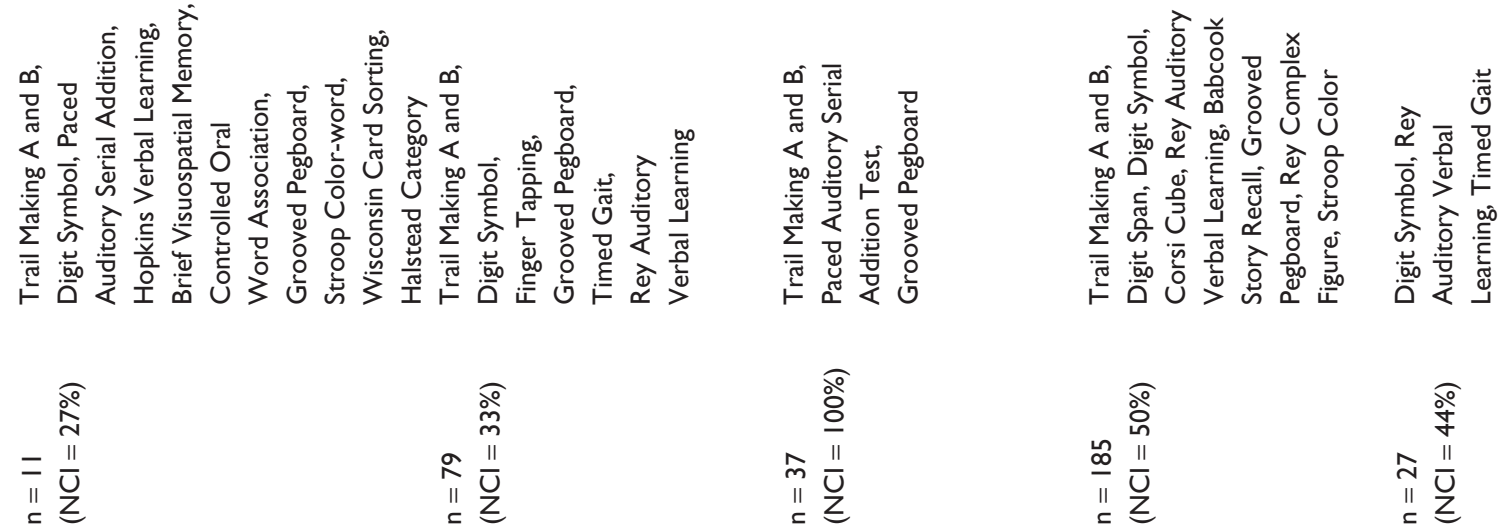

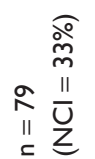

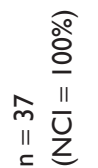

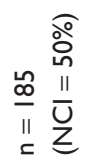

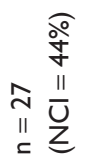

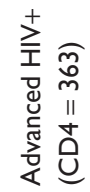

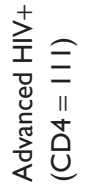

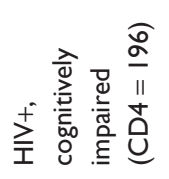

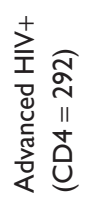

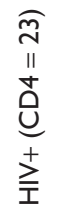
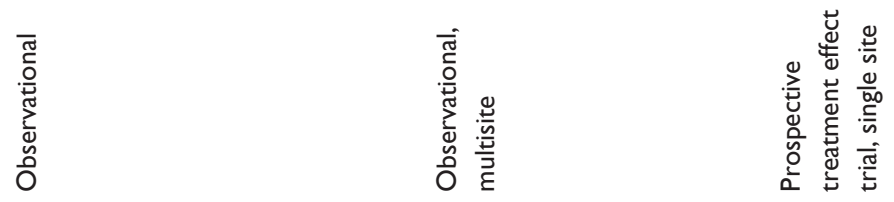

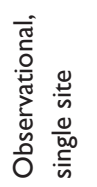

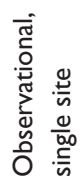

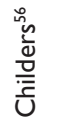

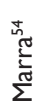

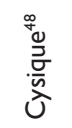

$\stackrel{\substack{N \\ \stackrel{N}{N}}}{\vdash}$

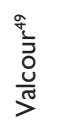




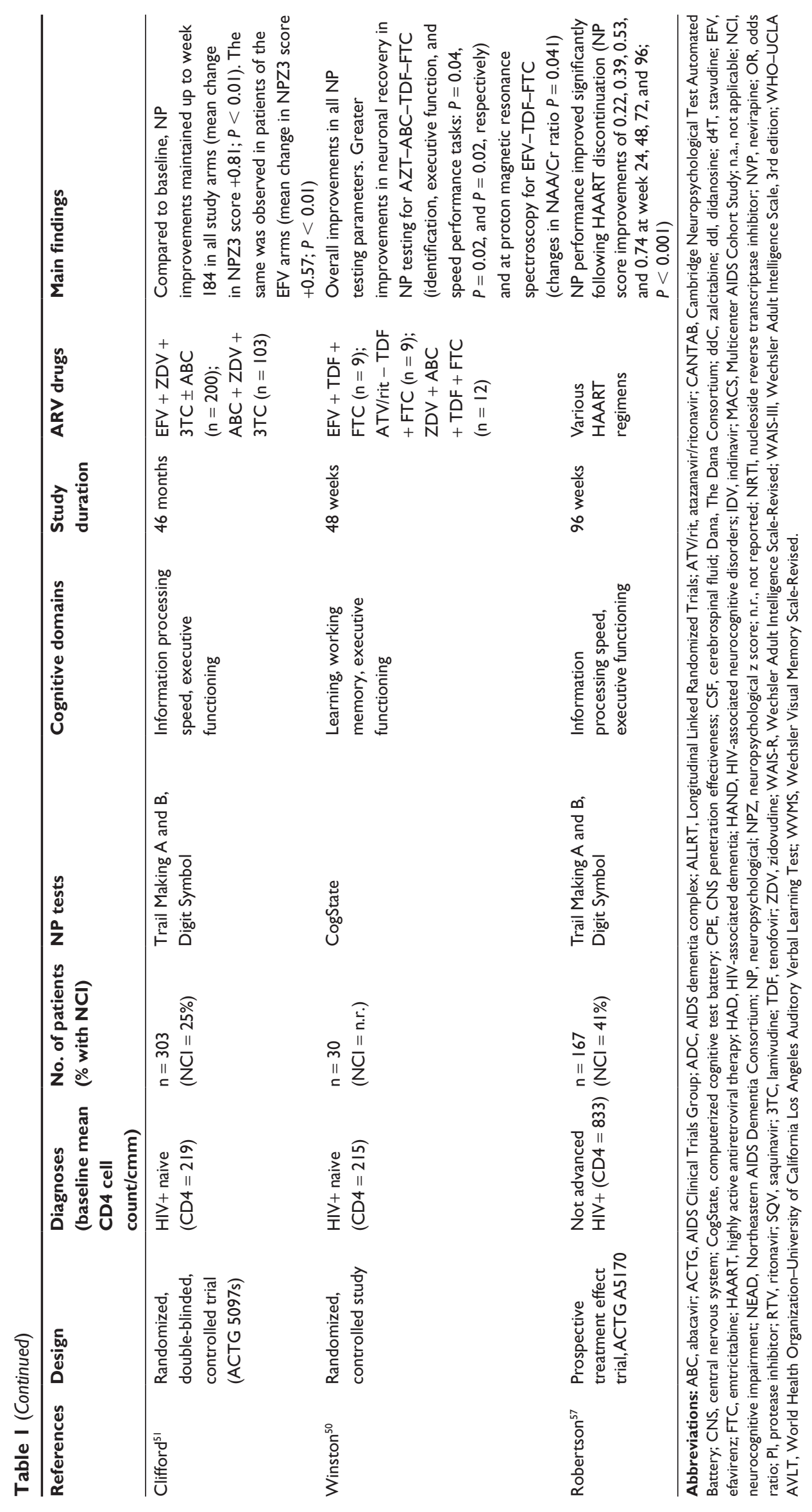


with longitudinal improvement in psychomotor speed, suggesting that subcortical brain structures mediating psychomotor speed might be sensitive not only to HIV-related neuropathological changes, but also to sustained benefits from HAART. ${ }^{33}$ Among participants in the MACS cohort monitored over a 5-year follow-up period, no decline in cognitive functions was reported. ${ }^{34}$ While confirming the overall improvements in several neurocognitive domains with HAART, Gibbie et al also reported a decline in depression scores. Interestingly, several interactions with depression scores were noted in this study since patients without depression improved significantly at cognitive testing while those with depression did not. ${ }^{22}$ Stable or improving cognition over a 2-year period was also reported in advanced HIVinfected patients. ${ }^{35}$ Within the randomized, double-blinded, controlled ACTG 5095 trial, a battery of three NP tests was administered to $303 \mathrm{ARV}$-naive patients randomly assigned to zidovudine-lamivudine-abacavir or zidovudine-lamivudineefavirenz or zidovudine-lamivudine-abacavir-efavirenz. NP measures were collected at baseline and at weeks 1, 4, 12, and 24. Symptoms that might be associated with efavirenz use (neurologic symptoms, sleep disorders, anxiety, and depression) were assessed by standardized questionnaires. Overall, NP performance, as measured by standardized NPZ3 scores, improved in all study arms, with greater changes occurring in the first week of treatment. Efavirenz use was associated with neurologic symptoms distinct from depression and anxiety. However, despite neurologic symptoms, improvement in NP performance was comparable in patients who were receiving efavirenz and those who were not. ${ }^{36}$

Improvement in psychomotor speed performance was also reported in association with systemic virologic suppression and decline in psychomotor speed performance with virologic rebound. ${ }^{37}$ However, the association of cognitive improvement with plasma HIV RNA decline was not confirmed in most subsequent studies. . $38,39^{-39}$

The notion that HAART is beneficial for cognitive functions was reinforced by prospective studies demonstrating that improvements in cognition were associated with HIV RNA decline in cerebrospinal fluid (CSF). Marra et al first reported the association of cognitive improvement with CSF viral load reductions. ${ }^{40}$ Similarly, significant improvement in neurologic and NP functioning and significant decline in CSF HIV RNA after HAART initiation or HAART change were reported. ${ }^{41}$ These observations were extended by Letendre et $\mathrm{al}^{39}$ who demonstrated that CSF HIV RNA suppression was an independent predictor of cognitive improvement. These data support the concept that inhibition of HIV replication within the CNS could be a critical step in arresting the neurodegenerative process.

\section{Variable benefits from HAART}

However, despite this success, accumulating evidences started to suggest that standard HAART regimens were often insufficient to fully reverse the cognitive dysfunction. A prospective observational study in advanced HIV-positive patients treated with HAART demonstrated that, aside from overall improvements in measures exploring different cognitive domains, more than $40 \%$ of cognitively impaired patients remained impaired despite more than 3 years of HAART. $^{42}$ Other authors reported similar findings in different populations of HIV-infected patients treated with HAART (Table 1). 22,33,35 These observations were supported by the analysis of two cohorts performed before and after the advent of HAART showing no difference in the overall occurrence of HIV dementia and NP abnormalities. ${ }^{13}$ Similarly, Cysique et al reported that the prevalence of NP impairment was not different across pre-HAART and HAART eras. ${ }^{43}$

In the HAART era, only a few randomized trials have been performed. A Phase III randomized, double-blinded, placebo-controlled trial of adding abacavir to optimal stable background therapy failed to find any differences in cognitive performance between the placebo and the abacavir group at week $12 .{ }^{44}$ This was in contrast with placebo-controlled trials performed in the monotherapy era.

To assess the extent to which cognitive functions changed overtime in patients with advanced disease, Cysique et al performed a prospective evaluation of 101 patients with CDC stage $\mathrm{C} 3$ disease. They found that although the majority of subjects improved, over the long term, $30 \%$ of patients showed reliable cognitive decline. Cognitive decline was related to lower nadir CD4 cell counts, past depressive episode, past HIV-related brain diseases, and number of previous AIDS-defining events. ${ }^{38}$ No improvement in neurocognitive status was reported in 14 naive patients with minor cognitive dysfunction treated with zidovudine-lamivudine-efavirenz, despite CSF and plasma HIV suppression. ${ }^{45}$ Moreover, a nonreversible component of cognitive impairment was noted in $63 \%$ of cognitively impaired patients treated with HAART for a mean of 63 months, with severity of cognitive impairment at enrolment being (at multivariable analyses) the strongest predictor of persistent impairment. ${ }^{46}$ These data confirmed that the NP benefits from HAART could be variable from patient to patient. Incidence and prevalence of neurocognitive impairment was examined among 1160 subjects within the ALLRT study. At least mild impairment 
was noted in $39 \%$ of patients with low (either current or nadir) CD4 cell counts being significantly associated with prevalent impairment. ${ }^{6}$ This suggests the presence of a nonreversible component of neural injury and that neural injury continues in some patients regardless of the success of ARV therapy. Studies demonstrating impaired cognitive functions despite HAART are supported by virological studies indicating that despite successful suppression of plasma viremia, HIV may replicate within the CNS at detectable levels and that detectable CSF HIV RNA may be associated with considerable levels of ARV drug resistance. ${ }^{47}$

Taken together, these data consistently indicate that current HAART regimens may be insufficient to fully treat and/or to fully prevent the development of HIV-associated cognitive impairment.

\section{Recent findings}

Recent achievements on ARV therapy and HAND expanded our knowledge and answered some important questions on the dynamics of cognitive changes, predictors of responses, and the outcome of different regimens.

Cysique et al performed a prospective study on patients with HAND retested with NP examinations at several targeted time points after HAART initiation. NP performance changes were standardized against comparison groups to account for practice effects. They found that rapid cognitive improvements could be observed in $13 \%$ of subjects within the first 12 weeks after HAART initiation or HAART change. The proportion of patients improving increased over time to $41 \%$ at week 48 . While providing the first evidence of short-term cognitive improvement as a marker of neurologic outcome of treatment, these findings also suggested that the window opportunity for NP improvement might be relatively long. ${ }^{48}$ Moreover, the extent of improvements was greater in patients with severe NP impairment at baseline, pointing to the reversibility of cognitive dysfunctions. Finally, the CNS penetration capacity was found to be the strongest predictor of cognitive improvement supporting the use of neuropenetrating ARV drugs to treat patients with HAND.

The SEARCH 001 Cohort Study was a prospective study to determine immunological and virological factors influencing cognition after first-time HAART in 15 individuals with HAD and in $15 \mathrm{HIV}$-infected patients without HAD. Variables were examined longitudinally to determine factors predictive of degree of cognitive recovery, and NP data of patients with HAD were compared to those of $230 \mathrm{HIV}$-negative controls to account for practice effects on repeated testing. Patients with HAD and patients without
HAD exhibited a robust cognitive response to HAART. Moreover, monocyte HIV DNA level correlates to cognitive performance before HAART and 48 weeks after HAART. The relationship between monocyte HIV DNA and the NP performance remained present in a multivariate model that included age, education, and baseline CD4 count. The authors concluded that baseline monocyte HIV DNA may predict 48-week cognitive performance. ${ }^{49}$ While confirming the improvement in NP performance after HAART initiation, these findings raise the possibility that short-term incomplete cognitive recovery with HAART may be a consequence of an active process related to HIV monocyte reservoir in the periphery.

The results of two randomized studies comparing different HAART regimens in measures of cerebral functioning tests were recently reported. Winston et al performed a randomized, controlled study of tenofovir-emtricitabine plus either efavirenz, or atazanavir-ritonavir or zidovudineabacavir on 30 treatment-naive patients. Cerebral function tests included neurocognitive testing and assessment of cerebral metabolites using proton magnetic resonance spectroscopy with calculations of $\mathrm{N}$-acetylaspartate-tocreatine $(\mathrm{NAA} / \mathrm{Cr})$ ratios in several anatomical voxels, at baseline and after 48 weeks. Overall, improvements in all neurocognitive testing parameters and increases of NAA/Cr ratios were observed during the study period and significant differences between treatment arms emerged. Over the 48 week of the study, greater improvements at cerebral metabolites assessment were observed for recipients of tenofoviremtricitabine plus efavirenz and greater improvements in neurocognitive function testing were observed for recipients of tenofovir-emtricitabine plus zidovudine-abacavir. The authors concluded that quadruple nucleoside reverse transcriptase inhibitor (NRTI) might have had superior positive effects on cognition as a consequence of optimal CNS drug penetration of these drugs and that NP improvements in the efavirenz arm might have been blunted by neuropsychiatric side effects of the drug. ${ }^{50}$ Finally, the results of the ACTG 5097s trial, a large, randomized, double-blind trial of 3 ARV regimens of zidovudine-lamivudine in combination with blinded efavirenz, abacavir, or efavirenz plus abacavir on long-term impact of efavirenz on NP performance, were recently published. ${ }^{51}$ A total of 117 patients were studied. Improvements were seen at week 184 in all groups compared to baseline performance $(P<0.01)$. Each of the three component tests (Trail Making A and B, Digit Symbol test) showed statistically significant improvements during the interval of the study in all study arms. Interestingly, although 
in efavirenz-based arms NP performance improvements were maintained over 3 years, higher efavirenz plasma levels were correlated with a small but significant negative impact on NPZ3 summary score $(r=-0.29 ; P<0.01)$.

\section{Data on cognition and ARV penetration into the CNS}

ARV drugs differ in their ability to cross blood-CNS interfaces and to reach the CNS at therapeutically effective levels. ARV drugs are considerably different not only in their CSF levels, but also in their capacity to reduce CSF HIV RNA to undetectable levels ${ }^{40,52}$ and improve cognition. ${ }^{8,24,50}$ The optimal treatment for HAND has not been established, but the general consensus is that the ability of ARV drugs to reach the CNS is a critical factor for patients' neurological response. ${ }^{9}$ Several ARV drugs, or drug combinations, have been shown to be more neurologically active than others on the basis of their capacity to suppress CSF viral load $\mathrm{d}^{40,52}$ and improve NP performance..$^{8,24,50}$ Moreover, it has been shown that virologic suppression in the CSF is associated with significant NP improvement. ${ }^{39-41}$ However, how to estimate the effectiveness of different ARV drug combinations in suppressing viral replication within the CNS and improving cognition remains controversial. Until recently, zidovudine, stavudine, abacavir, lamivudine, indinavir, efavirenz, and nevirapine were considered ARV drugs with good CNS effectiveness, ${ }^{9}$ generally on the bases of their CSF concentration from human studies. Recently, when trying to develop a clinically useful approach to estimating CNS effectiveness, a CNS-penetration-effectiveness (CPE) ranking system was proposed. This ranking system was based not only on CSF drug concentration, but also on the chemical properties of ARV drugs and clinical studies on drug ability to reduce CSF viral load and improve cognition. ${ }^{23,53}$ The authors found that HAART schemes with higher CPE ranks were associated with greater reductions of CSF HIV RNA levels. ${ }^{53}$ These data have been subsequently confirmed in independent studies. ${ }^{54}$ The ACTG 736 was a prospective study aimed to examine changes in CSF HIV RNA and in NP function in patients who begin or change an ARV regimen. A total of 79 patients were studied. Overall, participants took 48 different ARV regimens. Patients who were prescribed a regimen with $\mathrm{CPE}$ rank of at least 2 had significantly higher odds of suppression of CSF HIV RNA $(\mathrm{OR}=4.1 ; P=0.04) .{ }^{54}$

Whether or not higher CPE ranks are also associated with greater improvement in NP test has been recently investigated by different groups. Cysique et al performed a prospective evaluation of 101 patients with stage $\mathrm{C} 3$ disease. They found that neuroactive HAART regimens, defined according to the CPE rank, were associated with NP improvement. ${ }^{48}$ In a retrospective analysis of an observational cohort, Tozzi et al compared the CPE score and other scores that were used in previous published studies. The main outcome criterion was changes in cognition in five cognitive domains. Higher CPE scores, consistent with higher estimates of neuropenetration, were consistently and significantly associated with NP improvements, while higher estimates of neuropenetration with alternative scores were not. ${ }^{55}$ In contrast, Marra et al showed that ARV with good CNS penetration, as assessed by CPE rank, while more effective in reducing CSF viral load, were associated with poorer neurocognitive performance. Among 26 patients with impaired NP performance at entry, patients who were prescribed ARV regimens with $\mathrm{CPE}$ ranks of $<2(n=12)$ showed significant improvements in NPZ4 scores $(P=0.002)$, while patients who were prescribed regimens with a CPE rank of at least 2 showed no changes in NP measures. ${ }^{54}$ Among potential explanations of this unexpected finding, the authors mention the hypothesis of CNS toxicity of some ARV drugs or drug combinations. ${ }^{54}$

Thus, ARV regimens with estimated good CNS penetration seem more effective in controlling CSF viral replication. However, the question of whether drug combinations with better estimates of CNS penetration are associated with better cognition remains controversial and larger controlled trials are needed to address the topic.

\section{Data on structured treatment interruption}

Although no longer considered therapeutically beneficial, the neurocognitive effects of treatment interruption were examined in two independent studies. Childers et al reported that during structured treatment interruption (STI), NP performance did not change. Following reinitiation of HAART, improved viral suppression and immune restoration resulted in significant improvement in neurocognitive performance. ${ }^{56}$ In contrast, more recently, it has been reported that among asymptomatic patients who initiated HAART at CD4 counts $>350$ cells/uL, neurocognition improved significantly following ARV treatment discontinuation, suggesting a potential neurotoxicity associated with ARV drug use. ${ }^{57}$ Potential explanations of these inconsistent results include differences regarding degrees of immune function at baseline, nadir CD4 counts, and HAART regimens.

\section{Adjuvant therapies}

Aside from ARV therapy, ongoing work has focused on the potential role of adjuvant and neuroprotective therapies. 
However, although neuroprotective therapies might be needed over time, especially in patients not responding neurologically to HAART, their role appears to have unproven or limited benefits. The ability of minocycline, an antibiotic with potent anti-inflammatory and neuroprotective properties, to protect against HIV-associated neurodegeneration is supported mainly by studies on animal models. ${ }^{55}$ Recently, a placebo-controlled trial to assess the effectiveness of the selegiline transdermal system in patients with HIV-associated cognitive impairment failed to show any effect on cognitive performance and on HIV-induced metabolic brain injury as measured by proton magnetic resonance spectroscopy. ${ }^{59}$

\section{NP testing as an end point}

NP testing is routinely used to quantify the severity of cognitive impairment, define the pattern of involvement, and assess the impact of treatment. For these reasons, they have been included as an end point in most published studies on cognitive function in treated patients. However, regarding the use of NP testing as an end point, several questions remain. First, it is not easy to determine the potential role of practice effects because some studies did not include comparison groups; however, a consistent number of studies included methodological interventions to account for practice effects. ${ }^{8,24,25,33,48-50}$ Second, NP test batteries differ widely across studies. While a consistent number of studies utilized comprehensive NP batteries examining a relevant number of cognitive domains, some studies were performed with brief NP batteries exploring a limited range of NP functions, with limited sensitivity and specificity. Third, the presence of potential confounders, although carefully evaluated in most studies, was not universally examined.

With all the above mentioned limitations, NP testing should be included as an end point in all studies. Ideally, NP evaluation should be accompanied by careful assessment of confounders, structured neurological evaluation, assessment of depression, assessment of functional consequences of NP impairment, CSF examination with virological and biomarker studies, and functional neuroimaging.

\section{Discussion}

We have examined the existing literature on the effects of HAART on NP functions. A great number of studies on NP outcomes in patients receiving ARV therapy have been published to date. However, several methodological and statistical issues should be considered in evaluating the existing literature. The strength of the evidences of studies reported in this review varies widely and a hierarchy of evidences exists. Small study size and lack of adequate statistical power could represent important limitations of some studies, especially those with a cross-sectional design. In general, observational cohorts may provide more information, but observational studies tend to include patients who are already on HAART and examine NP end points at nonplanned intervals. Unfortunately, most published studies are either cross-sectional or observational in design. Some carefully planned prospective treatment effect cohorts have also been conducted and provided a consistent body of knowledge on the topic. However, only a few randomized, controlled studies have been performed to date, making it difficult to draw definitive conclusions on some aspects. Thus, differences between study designs may account for a number of discrepancies between results.

Moreover, there are also remarkable differences among patients' characteristics. Only a few studies examined exclusively patients with documented cognitive impairment. Some studies examined patients with advanced diseases including subjects with normal cognition. Other studies enrolled neurologically asymptomatic patients at an early disease stage and with high CD4 cell counts. ARV therapy schemes and treatment duration also varied considerably. The presence and nature of comorbid conditions were not consistently examined in all studies. Finally, nature and length of NP batteries varied broadly, making NP test results difficult to compare.

With all the above-mentioned considerations, it is rather clear that most studies indicate that use of HAART results in improvement of neurocognitive functions. Two randomized clinical trials clearly showed the superiority of zidovudine monotherapy over placebo in improving cognition both in advanced HIV-infected patients ${ }^{24}$ and in patients with HAD. ${ }^{25}$ A randomized trial performed in the early HAART era showed that zidovudine-didanosine-nevirapine therapy improved NP performance compared to dual NRTI therapy. ${ }^{8}$ Recently, in a randomized, controlled study, greater improvement in neurocognitive function testing was reported in naive patients treated with tenofovir-emtricitabine-zidovudineabacavir compared to tenofovir-emtricitabine-atazanavirritonavir. ${ }^{50}$ Finally, the randomized, double-blind trial ACTG 5097s showed statistically significant improvements over 3 years of study duration in all study arms. ${ }^{51}$ Most cohort studies also indicate that use of HAART is associated with improved cognition. 3,6,26-35,37,38,40-42,45,46,48,49,55,56 Interestingly, almost all randomized studies ${ }^{8,24,25,36,50,51}$ and carefully designed prospective cohorts on advanced and/or cognitively impaired patients ${ }^{30,31,37,40,41,48}$ reported improvements 
in cognition with HAART use. Improvements were more pronounced in severely cognitively impaired subjects ${ }^{48}$ and occur within a few weeks of ARV therapy initiation or change. ${ }^{24,25,36,48,51}$ These data clearly indicate that there is a reversible component of HIV-associated cognitive impairment linked to HAART-induced reduction of viral replication within the CNS. ${ }^{39-41}$

However, a great variability of treatment responses was reported since improvements in NP functions were neither full ${ }^{6,38,42,43,45,46,55}$ nor universal. ${ }^{54,57}$ It is evident that a consistent proportion of patients failed to fully revert the cognitive dysfunction. Persistent NP impairment despite HAART was reported in a consistent number of patients. ${ }^{646}$ The severity of NP impairment ${ }^{46}$ and previous advanced immunosuppression ${ }^{6}$ were reported as associated with persistent impairment. The presence of a nonreversible component of NCI suggests the presence of nonreversible neural injury that presumably derives from residual damage secondary to neurotoxic cascades within the CNS. In fact, in vivo cognitive impairment has been associated with postmortem neuropathologic changes ${ }^{60}$ and with in vivo evidences at magnetic resonance imaging studies of white matter tract injury consistent with axonal or neuronal $\operatorname{loss}^{61}$ and gray and white matter brain loss. ${ }^{62}$

Other potential explanations of persistent cognitive impairment in patients treated with successful HAART include ongoing viral replication of compartmentalized HIV within the $\mathrm{CNS}^{47}$ and insufficient penetration of some ARV drugs within the CNS. ${ }^{9}$ Moreover, ongoing chronic inflammatory processes within the CNS and vascular factors might also contribute to persistent cognitive impairment in patients treated with HAART. Risk factors for cardiovascular disease (ie, smoking, hypertension, dyslipidemia, obesity, and diabetes) contribute to the pathogenesis of both vascular dementia and Alzheimer disease. ${ }^{63,64}$ Cardiovascular risk factors ${ }^{21}$ and insulin resistance ${ }^{19}$ are also associated with cognitive impairment in HIV-infected patients. Raised inflammatory markers are common to cardiovascular disease,${ }^{65}$ metabolic syndrome, ${ }^{66}$ dementia, ${ }^{67}$ and accelerated aging in HIV-infected patients. ${ }^{68}$ Recently, increased plasma levels of markers of vascular dysfunction have been associated with impairment in verbal comprehension and perceptual reasoning in perinatally HIV-infected youth. ${ }^{69}$ High levels of immune activation markers, like $\beta$-2-microglobuline, neopterine, quinolonic acid, and monocyte chemoattractant protein-1 (MCP-1) within the $\mathrm{CSF}$ are frequently found in patients with $\mathrm{HAD}^{70}$ Persistent intrathecal immune activation was described in HIV-infected patients on ARV therapy having CSF HIV RNA between 2.5 and 49 copies $/ \mathrm{mL}^{71}$ The same was reported in patients successfully treated with HAART for more than 4 years. ${ }^{72}$ Taken together, these data suggest that inflammatory mediators released within the CNS could initiate and maintain the neurodegenerative process resulting in neuronal dysfunction and death in patients treated with HAART. ${ }^{73}$

Finally, a few studies indicate no changes in neurocognitive performance or even NP deterioration with HAART use, suggesting potential toxicity from ARV drug use. One report observed no changes in NPZ4 scores in patients receiving neuropenetrating ARV drugs and significant improvements in patients receiving ARV combination with lower estimate of CNS penetration. ${ }^{54} \mathrm{~A}$ second article reported NP improvement after HAART discontinuation. ${ }^{57}$ However, the first study had a relatively small number of patients, and the second included a very short battery of NP tests. Moreover, both studies have not been confirmed. However, although evidence of CNS ARV toxicity is relatively low, the concern is rather reasonable, and randomized, controlled trials on the topic are needed.

In conclusion, remarkable progress has been made on our understanding prevalence, risk factors, and response to treatment of HIV-associated cognitive disturbances. However, a number of open questions and unresolved clinical needs still remain. There is a need for randomized prospective trials and carefully planned large prospective cohort studies to answer unresolved issues.

\section{Acknowledgment}

This study was partially supported by the Italian Ministry of Health, I.R.C.C.S 'Ricerca Corrente'.

\section{Disclosure}

The authors report no conflicts of interest in this work.

\section{References}

1. Antinori A, Arendt G, Becker JT, et al. Updated research nosology for HIV-associated neurocognitive disorders. Neurology. 2007;69(18): 1789-1799.

2. Dore GJ, Correll PK, Li Y, Kaldor JM, Cooper DA, Brew BJ. Changes to AIDS dementia complex in the era of highly active antiretroviral therapy. AIDS. 1999;13(10):1249-1253.

3. Sacktor N, Lyles RH, Skolasky R, et al. HIV-associated neurologic disease incidence changes: Multicenter AIDS Cohort Study, 1990-1998. Neurology. 2001;56(2):257-260.

4. D'Arminio Monforte A, Cinque P, Mocroft A, et al. Changing incidence of central nervous system diseases in the EuroSIDA cohort. Ann Neurol. 2004;55(3):320-328.

5. Ferrando S, van Gorp W, McElhiney M, Goggin K, Sewell M, Rabkin J. Highly active antiretroviral treatment in HIV infection: benefits for neuropsychological function. AIDS. 1998;12(8):F65-F70.

6. Robertson KR, Smurzynski M, Parsons TD, et al. The prevalence and incidence of neurocognitive impairment in the HAART era. AIDS. 2007; 21(14):1915-1921. 
7. Simioni S, Cavassini M, Annoni J-M, et al. Cognitive dysfunction in HIV patients despite long-standing suppression of viremia. AIDS. 2010;24(9):1243-1250.

8. Price RW, Yiannoutsos CT, Clifford DB, et al. Neurological outcomes in late HIV infection: adverse impact of neurological impairment on survival and protective effect of antiviral therapy. AIDS Clinical Trial Group and Neurological AIDS Research Consortium study team. AIDS. 1999;13(13):1677-1685.

9. McArthur JC, Brew BJ, Nath A. Neurological complications of HIV infection. Lancet Neurol. 2005;4(9):543-555.

10. Tozzi V, Balestra P, Murri R, et al. Neurocognitive impairment influences quality of life in HIV-infected patients receiving HAART. Int J STD AIDS. 2004;15(4):254-259.

11. Gorman AA, Foley JM, Ettenhofer ML, Hinkin CH, van Gorp WG. Functional consequences of HIV-associated neuropsychological impairment. Neuropsychol Rev. 2009;19(2):186-203.

12. Valcour VG, Shikuma CM, Watters MR, Sacktor NC. Cognitive impairment in older HIV-1-seropositive individuals: prevalence and potential mechanisms. AIDS. 2004;18 Suppl 1:S79-S86.

13. Sacktor N, McDermott MP, Marder K, et al. HIV-associated cognitive impairment before and after the advent of combination therapy. J Neurovirol. 2002;8(2):136-142.

14. Dore GJ, McDonald A, Li Y, Kaldor JM, Brew BJ; National HIV Surveillance Committee. Marked improvement in survival following AIDS dementia complex in the era of highly active antiretroviral therapy. AIDS. 2003;17(10):1539-1545.

15. Valcour V, Shikuma C, Shiramizu B, et al. Higher frequency of dementia in older HIV-1 individuals: the Hawaii aging with HIV-1 cohort. Neurology. 2004;63(5):822-827.

16. Wong MH, Robertson K, Nakasujja N, et al. Frequency of and risk factors for HIV dementia in a HIV clinic in sub-Saharan Africa. Neurology. 2007;68(5):350-355.

17. Munoz-Moreno JA, Fumaz CR, Ferrer MJ, et al. Nadir CD4 cell counts predicts neurocognitive impairment in HIV-infected patients. AIDS Res Hum Retroviruses. 2008;24(10):1301-1307.

18. Tozzi V, Balestra P, Lorenzini P, et al. Prevalence and risk factors for human immunodeficiency virus-associated neurocognitive impairment, 1992-2002: results from an urban observational cohort. J Neurovirol. 2005;11(3):265-273.

19. Valcour VG, Sacktor NC, Paul RH, et al. Insulin resistance is associated with cognition among HIV-1-infected patients: the Hawaii aging with HIV cohort. J Acquir Immune Defic Syndr. 2006;43(4):405-410.

20. Ryan EL, Morgello S, Isaacs K, Naseer M, Gerits P; Manhattan HIV Brain Bank. Neuropsychiatric impact of hepatitis C on advanced HIV. Neurology. 2004;62(6):957-962.

21. Wright EJ, Grund B, Robertson K; INSIGHT SMART Study Group. Cardiovascular risk factors associated with lower baseline cognitive performance in HIV-positive persons. Neurology. 2010;75(10):864-873.

22. Gibbie T, Mijch A, Ellen S, et al. Depression and neurocognitive performance in individuals with HIV/AIDS: 2-year follow-up. HIV Med. 2006;7(2):112-121.

23. Clifford DB. HIV-associated neurocognitive disease continues in the antiretroviral era. Top HIV Med. 2008;16(2):94-98.

24. Schmitt FA, Bigley JW, McKinnis R, Logue PE, Evans RW, Drucker JL. Neuropsychological outcome of zidovudine (AZT) treatment of patients with AIDS and AIDS-related complex. N Engl J Med. 1988;319(24): 1573-1578.

25. Sidtis JJ, Gatsonis C, Price RW, et al. Zidovudine treatment of the AIDS dementia complex: results of a placebo-controlled trial. AIDS Clinical Trials Group. Ann Neurol. 1993;33(4):343-349.

26. Tozzi V, Narciso P, Galgani S, et al. Effects of zidovudine in 30 patients with mild to end-stage AIDS dementia complex. AIDS. 1993;7(5):683-692.

27. Tozzi V, Balestra P, Galgani S, et al. Positive and sustained effects of highly active antiretroviral therapy on HIV 1 - associated neurocognitive impairment. AIDS. 1999;13(14):1889-1897.
28. Sacktor NC, Lyles RH, Skolasky RL, et al. Combination antiretroviral therapy improves psychomotor speed performance in HIV-seropositive homosexual men. Multicenter AIDS Cohort Study (MACS). Neurology. 1999;52(8):1640-1647.

29. Cohen RA, Boland R, Paul R, et al. Neurocognitive performance enhanced by highly active antiretroviral therapy in HIV-infected women. AIDS. 2001;15(3):341-345.

30. Richardson JL, Martin EM, Jimenez N, et al. Neuropsychological functioning in a cohort of HIV infected women: importance of antiretroviral therapy. J Int Neuropsychol Soc. 2002;8(6):781-793. Erratum in: J Int Neuropsychol Soc. 2005;11(1):120.

31. Sacktor N, Nakasujja N, Skolasky R, et al. Antiretroviral therapy improves cognitive impairment in HIV+ individuals in sub-Saharan Africa. Neurology. 2006;67(2):311-314.

32. Suarez S, Baril L, Stankoff B, et al. Outcome of patients with HIV-1related cognitive impairment on highly active antiretroviral therapy. AIDS. 2001;15(2):195-200.

33. Ferrando SJ, Rabkin JG, van Gorp W, Lin SH, McElhiney M. Longitudinal improvement in psychomotor processing speed is associated with potent combination antiretroviral therapy in HIV-1 infection. $J$ Neuropsychiatry Clin Neurosci. 2003;15(2):208-214.

34. Cole MA, Margolick JB, Cox C, et al; Multicenter AIDS Cohort Study. Longitudinally preserved psychomotor performance in long-term asymptomatic HIV-infected individuals. Neurology. 2007;69(24): 2213-2220.

35. McCutchan JA, Wu JW, Robertson K, et al. HIV suppression by HAART preserves cognitive function in advanced, immune-reconstituted AIDS patients. AIDS. 2007;21(9):1109-1117.

36. Clifford DB, Evans S, Yang Y, et al. Impact of efavirenz on neuropsychological performance and symptoms in HIV-infected individuals. Ann Intern Med. 2005;143(10):714-721.

37. Sacktor N, Skolasky RL, Tarwater PM, et al; Multicenter AIDS Cohort Study (MACS). Response to systemic HIV viral load suppression correlates with psychomotor speed performance. Neurology. 2003;61(4): 567-569.

38. Cysique LA, Maruff P, Brew BJ. Variable benefit in neuropsychological function in HIV-infected HAART-treated patients. Neurology. 2006; 66(9):1447-1450.

39. Letendre SL, McCutchan JA, Childers ME, et al. Enhancing antiretroviral therapy for human immunodeficiency virus cognitive disorders. Ann Neurol. 2004;56(3):416-423.

40. Marra CM, Lockhart MS, Zunt JR, Perrin M, Coombs RW, Collier AC. Changes in CSF and plasma HIV-1 RNA and cognition after starting potent antiretroviral therapy. Neurology. 2003;60(8):1388-1390.

41. Robertson KR, Robertson WT, Ford S, et al. Highly active antiretroviral therapy improves neurocognitive functioning. J Acquir Immune Defic Syndr. 2004;36(1):562-566.

42. Tozzi V, Balestra P, Galgani S, et al. Changes in neurocognitive performance in a cohort of patients treated with HAART for 3 years. J Acquir Immune Defic Syndr. 2001;28(1):19-27.

43. Cysique LA, Maruff P, Brew BJ. Prevalence and pattern of neuropsychological impairment in human immunodeficiency virus-infected/ acquired immunodeficiency syndrome (HIV/AIDS) patients across pre- and post-highly active antiretroviral therapy eras: a combined study of two cohorts. J Neurovirol. 2004;10(6):350-357.

44. Brew BJ, Halman M, Catalan J, et al. Factors in AIDS dementia complex trial design: results and lessons from the abacavir trial. PLoS Clin Trials. 2007;2(3):e13.

45. Carvalhal AS, Rourke SB, Belmonte-Abreu P, Correa J, Goldani LZ. Evaluation of neuropsychological performance of HIV-infected patients with minor motor cognitive dysfunction treated with highly active antiretroviral therapy. Infection. 2006;34(6):357-360.

46. Tozzi V, Balestra P, Bellagamba R, et al. Persistence of neuropsychologic deficits despite long-term highly active antiretroviral therapy in patients with HIV-related neurocognitive impairment: prevalence and risk factors. J Acquir Immune Defic Syndr. 2007;45(2):174-182. 
47. Canestri A, Lescure FX, Jaureguiberry S, et al. Discordance between cerebral spinal fluid and plasma HIV replication in patients with neurological symptoms who are receiving suppressive antiretroviral therapy. Clin Infect Dis. 2010;50(5):773-778.

48. Cysique LA, Vaida F, Letendre S, et al. Dynamics of cognitive change in impaired HIV-positive patients initiating antiretroviral therapy. Neurology. 2009;73(5):342-348.

49. Valcour VG, Shiramizu BT, Sithinamsuwan P, et al; Southeast Asia Research Collaboration with the University of Hawaii 001 protocol team. HIV DNA and cognition in a Thai longitudinal HAART initiation cohort: the SEARCH 001 Cohort Study. Neurology. 2009;72(11):992-998.

50. Winston A, Duncombe C, Li PC, et al. Does choice of combination antiretroviral therapy (cART) alter changes in cerebral function testing after 48 weeks in treatment-naive, HIV-1-infected individuals commencing cART? A randomized, controlled study. Clin Infect Dis. 2010;50(6): 920-929.

51. Clifford DB, Evans S, Yang Y, Acosta EP, Ribaudo H, Gulick R; A5097s Study Team. Long-term impact of efavirenz on neuropsychological performance and symptoms in HIV-infected individuals (ACTG 5097s). HIV Clin Trials. 2009;10(6):343-355.

52. Polis MA, Suzman DL, Yoder CP, et al. Suppression of cerebrospinal fluid HIV burden in antiretroviral naive patients on a potent four-drug antiretroviral regimen. AIDS. 2003;17(8):1167-1172.

53. Letendre S, Marquie-Beck J, Capparelli E, et al. Validation of the CNS penetration-effectiveness rank for quantifying antiretroviral penetration into the central nervous system. Arch Neurol. 2008;65(1):65-70.

54. Marra CM, Zhao Y, Clifford DB, et al. Impact of combination antiretroviral therapy on cerebrospinal fluid HIV RNA and neurocognitive performance. AIDS. 2009;23(11):1359-1366.

55. Tozzi V, Balestra P, Salvatori MF, et al. Changes in cognition during antiretroviral therapy: comparison of 2 different ranking systems to measure antiretroviral drug efficacy on HIV-associated neurocognitive disorders. J Acquir Immune Defic Syndr. 2009;52(1):56-63. Erratum in: J Acquir Immune Defic Syndr. 2009;52(4):529.

56. Childers ME, Woods SP, Letendre S, et al; San Diego HIV Neurobehavioral Research Center Group. Cognitive functioning during highly active antiretroviral therapy interruption in human immunodeficiency virus type 1 infection. J Neurovirol. 2008;14(6):550-557.

57. Robertson KR, Su Z, Margolis DM, et al; A5170 Study Team. Neurocognitive effects of treatment interruption in stable HIV-positive patients in an observational cohort. Neurology. 2010;74(16):1260-1266.

58. Zink MC, Uhrlaub J, DeWitt J, et al. Neuroprotective and antihuman immunodeficiency virus activity of minocycline. JAMA. 2005; 293(16):2003-2011.

59. Schifitto G, Yiannoutsos CT, Ernst T, et al; ACTG 5114 Team. Selegiline and oxidative stress in HIV-associated cognitive impairment. Neurology. 2009;73(23):1975-1981.
60. Moore DJ, Masliah E, Rippeth JD, et al; HNRC Group. Cortical and subcortical neurodegeneration is associated with HIV neurocognitive impairment. AIDS. 2006;20(6):879-887.

61. Gongvatana A, Schweinsburg BC, Taylor MJ, et al. White matter tract injury and cognitive impairment in human immunodeficiency virusinfected individuals. J Neurovirol. 2009;15(2):187-195.

62. Cardenas VA, Meyerhoff DJ, Studholme C, et al. Evidence for ongoing brain injury in human immunodeficiency virus-positive patients treated with antiretroviral therapy. J Neurovirol. 2009;15(4):324-333.

63. Hayden KM, Zandi PP, Lyketsos CG, et al; Cache County Investigators. Vascular risk factors for incident Alzheimer disease and vascular dementia: the Cache County study. Alzheimer Dis Assoc Disord. 2006; 20(2):93-100.

64. Anstey KJ, Lipnicki DM, Low LF. Cholesterol as a risk factor for dementia and cognitive decline: a systematic review of prospective studies with meta-analysis. Am J Geriatr Psychiatry. 2008;16(5):343-354.

65. Sattar N, Murray HM, Welsh P, et al; Prospective Study of Pravastatin in the Elderly at Risk (PROSPER) Study Group. Are markers of inflammation more strongly associated with risk for fatal than for nonfatal vascular events? PLoS Med. 2009;6(6):e1000099. Epub 2009 Jun 23.

66. Kowalska I, Straczkowski M, Nikolajuk A, et al. Insulin resistance, serum adiponectin, and proinflammatory markers in young subjects with the metabolic syndrome. Metabolism. 2008;57(11): 1539-1544.

67. Engelhart MJ, Geerlings MI, Meijer J, et al. Inflammatory proteins in plasma and the risk of dementia: the Rotterdam study. Arch Neurol. 2004;61(5):668-672.

68. Deeks SG. Immune dysfunction, inflammation, and accelerated aging in patients on antiretroviral therapy. Top HIV Med. 2009;17(4): $118-123$.

69. Kapetanovic S, Leister E, Nichols S, et al; Pediatric HIV/AIDS cohort study Team. Relationships between markers of vascular dysfunction and neurodevelopmental outcomes in perinatally HIV-infected youth. AIDS. 2010;24(10):1481-1491.

70. Price RW, Epstein LG, Becker JT, et al. Biomarkers of HIV-1 CNS infection and injury. Neurology. 2007;69(18):1781-1788.

71. Yilmaz A, Price RW, Spudich S, et al. Persistent intrathecal immune activation in HIV-1-infected individuals on antiretroviral therapy. J Acquir Immune Defic Syndr. 2008;47(2):168-173.

72. Edén A, Price RW, Spudich S, et al. Immune activation of the central nervous system is still present after $>4$ years of effective highly active antiretroviral therapy. J Infect Dis. 2007;196(12):1779-1783.

73. Kaul M, Lipton SA. Mechanisms of neuroimmunity and neurodegeneration associated with HIV-1 infection and AIDS. J Neuroimmune Pharmacol. 2006;1(2):138-151.
Neurobehavioral HIV Medicine

\section{Publish your work in this journal}

Neurobehavioral HIV Medicine is an international, peer-reviewed, open access journal focusing on advances in research in HIV/ AIDS, with specific reference to the neurological, psychiatric and behavioral consequences of the disease, concomitant infections and specific antiretroviral therapy. The manuscript

\section{Dovepress}

management system is completely online and includes a very quick and fair peer-review system, which is all easy to use. Visit http://www.dovepress.com/testimonials.php to read real quotes from published authors. 